\title{
Effects of Somatothermal Far-Infrared Ray on Primary Dysmenorrhea: A Pilot Study
}

\author{
Yu-Min Ke, ${ }^{1}$ Ming-Chiu Ou, ${ }^{2}$ Cheng-Kun Ho, ${ }^{2}$ Yung-Sheng Lin, ${ }^{2}$ \\ Ho-Yen Liu, ${ }^{2}$ and Wen-An Chang ${ }^{2}$ \\ ${ }^{1}$ Department of Obstetrics and Gynecology, Taichung Veterans General Hospital, Taichung 40705, Taiwan \\ ${ }^{2}$ Department of Applied Cosmetology and Master Program of Cosmetic Science, Hungkuang University, Taichung 43302, Taiwan
}

Correspondence should be addressed to Yung-Sheng Lin, linys@sunrise.hk.edu.tw

Received 27 June 2012; Revised 26 November 2012; Accepted 29 November 2012

Academic Editor: R. Govindarajan

Copyright (c) 2012 Yu-Min Ke et al. This is an open access article distributed under the Creative Commons Attribution License, which permits unrestricted use, distribution, and reproduction in any medium, provided the original work is properly cited.

The purpose of this study was to assess the beneficial effects of using a far-infrared (FIR) belt on the management of patients with primary dysmenorrhea. This is the first study to determine the efficacy of somatothermal FIR using a parallel-arm randomized sham-controlled and double-blinded design with objective physical evidence and psychometric self-reports. Fifty-one Taiwanese women with primary dysmenorrhea were enrolled in the study. Results indicate that there was an increased abdominal temperature of $0.6^{\circ} \mathrm{C}$ and a $3.27 \%$ increase in abdominal blood flow in the FIR group (wearing FIR belt) compared to those in the control group (wearing sham belt). Verbal rating scale and numeric rating scale scores in the FIR group were both lower than those in the control group. Compared to the blank group (wearing no belt), the average dysmenorrhea pain duration of the FIR group was significantly reduced from 2.5 to 1.8 days, but there was no significant difference in the control group. These results demonstrate that the use of a belt made of far-infrared ceramic materials can reduce primary dysmenorrhea.

\section{Introduction}

Dysmenorrhea is pain with menstruation, which may be accompanied by headache, dizziness, nausea, vomiting, diarrhea, cold sweats, or other symptoms $[1,2]$. For clinical purposes, dysmenorrhea is classified into primary and secondary dysmenorrhea. Previous studies showed that $50 \%$ to $80 \%$ of women worldwide have experienced dysmenorrhea, and the majority of these women are teenagers and have primary dysmenorrhea [2, 3]. Primary dysmenorrhea, a condition associated with ovulatory cycles, is the occurrence of menstrual cramps in the absence of demonstrable disease that could account for symptoms [4]. After the onset of menstruation, pain typically starts within hours peaking between 12 and 24 hours and persisting for 12-72 hours.

Clinical research has shown that the main cause of primary dysmenorrhea may be associated with increased production of endometrial prostaglandin, resulting in a high concentration of prostaglandins in blood. The painrelated biomolecular induction of cyclooxygenase (COX-2) and prostaglandin is strongly associated with the severity of primary dysmenorrhea [4]. Excessive prostaglandin causes uterine contractions, ischemia, cramping, and pelvic pain. Nonsteroidal anti-inflammatory drugs (NSAIDs) are commonly used in women with dysmenorrhea, because they can inhibit prostaglandin synthesis and effectively alleviate the symptoms of dysmenorrhea. However, NSAIDs may lead to many adverse effects, including indigestion, headache, and drowsiness, and their rate of failure to reduce dysmenorrhea may reach $20 \%$ [5]. Therefore, complementary and alternative medicine (CAM) is becoming an increasingly popular choice for alleviation of dysmenorrhea [6-13].

For women with dysmenorrhea, the application of local heat can reduce muscle tension and relax abdominal muscles to reduce pain caused by muscle spasms. Heat can increase pelvic blood circulation to eliminate local blood and body fluid retention and diminish congestion and swelling, thereby enabling a reduction in pain caused by nerve compression. Previous studies reported that local heat inhibits pain signals and increases proprioception $[14,15]$. 
Heating pads and hot water have long been used as a folk remedy for the treatment of dysmenorrhea [14]. Akin et al. [14] reported that a heating patch on the abdomen can be as effective as ibuprofen to treat dysmenorrhea. However, external energy is needed to provide the heat, and there is a risk of burn accident with improper temperature control. In addition, there are a number of disadvantages using complementary and alternative medicine therapies, such as Chinese herbal medicine [12], acupuncture [16], acupressure $[7,17]$, aromatherapy $[8]$, and transcutaneous electrical nerve stimulation [11], which may include inconvenience of application and energy consumption, among others.

Far-infrared (FIR) rays are invisible electromagnetic waves, which can produce many biological effects including thermal and nonthermal effects [18-23]. When FIR materials are used to cover the skin, it can reduce body heat loss owing to their thermal insulation properties [24-26]. FIR can promote microcirculation, accelerate wound healing, modulate sleep, and treat depression [27-29]. In general, as an alternative nonpharmacologic therapy, FIR has been shown to be an effective and safe modality for promoting health in patients with various medical conditions.

The use of electrothermal FIR belts at a temperature of $50^{\circ} \mathrm{C}$ was recently reported to be an effective treatment for primary dysmenorrhea $[30,31]$. The most common adverse event was burn caused by the incorrect use of the hot pack [30]. While a number of studies have investigated the therapeutic effects of electrothermal FIR belts, to our knowledge the health benefits of somatothermal FIR belts have not been previously reported in the literature. Thus, the aim of the present study was to test the effectiveness of a somatothermal FIR belt as a novel alternative therapy for the relief of primary dysmenorrhea.

\section{Materials and Methods}

2.1. Participants. The clinical trial was approved by the institutional review board (IRB) at HungKuang University, (Project number 98-B-002). The participants were screened by a gynecologist. Inclusion criteria were age 18 years or older which can legally give consent to participate in this clinical trial and history of painful menstruation for at least one year. Physical examination and ultrasonography were used to support diagnosis of primary dysmenorrhea. Patients who had gynecological diseases or who used other FIR products within one month before the study were excluded. Patients were instructed not to use any type of medication, such as NSAIDs or oral pills, to relieve painful menstruation during the study period. In the last analysis, a total of 51 subjects aged 19 to 35 years were enrolled in this study. The investigation was conducted between January 1, 2010 and December 31, 2010.

2.2. Belts. Two kinds of belts each measuring $15 \mathrm{~cm} \times$ $70 \mathrm{~cm}$ were used in this study. The FIR belt and the control belt were embedded with and without $10 \mathrm{wt} \%$ FIR ceramic powders, respectively. As previously reported [1923], the FIR ceramic powders were composed of numerous

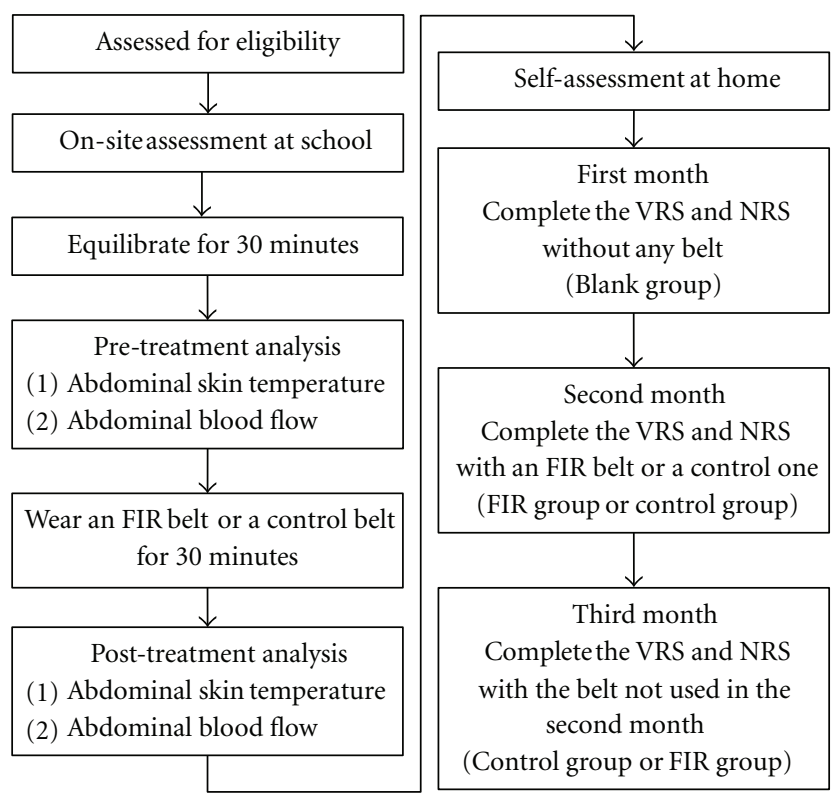

FIgURE 1: The flow chart of participants through the trial.

mineral oxides, including aluminum oxide, ferric oxide, magnesium oxide, and calcium carbonate. The FIR energy, at wavelengths between 3 and $16 \mu \mathrm{m}$, was $10.16 \mathrm{~mW} / \mathrm{cm}^{2}$ as determined using an SR5000 spectroradiometer (CI, Ltd., Migdal HaEmek, Israel) at the Industrial Technology Research Institute, Taiwan.

2.3. Abdominal Temperature and Blood Flow. All measurements and procedures in this study were performed in a climate-controlled room at a constant temperature and humidity $\left(23^{\circ} \mathrm{C}\right.$ and $60 \%$ relative humidity) [32]. Before assessment, participants were required to lie down in the aforementioned room for 30 minutes to acclimatize to the indoor climatic conditions. Participants were thermographed to measure abdominal temperatures using a Fluke Ti25 thermal imager (Fluke Corporation, Everett, WA, USA), and their abdominal blood flows were detected using a MoorLDI2-IR Laser Doppler Imager (Moor Instruments Ltd., Devon, UK) as described in the previous report [31]. Subsequently, a double-blind method was used to randomly assign subjects to the FIR belt $(n=26)$ group or control belt $(n=25)$ group. Each subject then wore the belt around the abdominal region for 30 minutes. After removing the belt, posttreatment measurements of abdominal temperatures and blood flows were performed.

2.4. Menstrual Pain Assessment. All participants $(n=51)$ completed two questionnaires, the verbal rating scale (VRS) and the numeric rating scale (NRS) [33, 34], to assess the extent of pain on days 1 to 3 of three menstrual cycles. As shown in Figure 1, the pain assessment for the first menstrual cycle was conducted without any belt (blank group). For the second and third menstrual cycles, each participant was instructed to wear the belt on the abdominal region for 


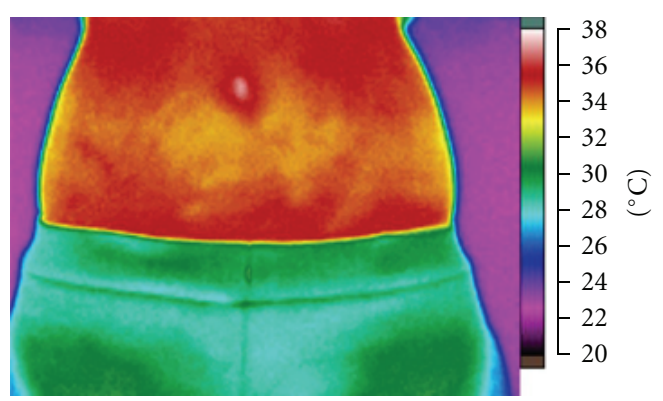

(a)

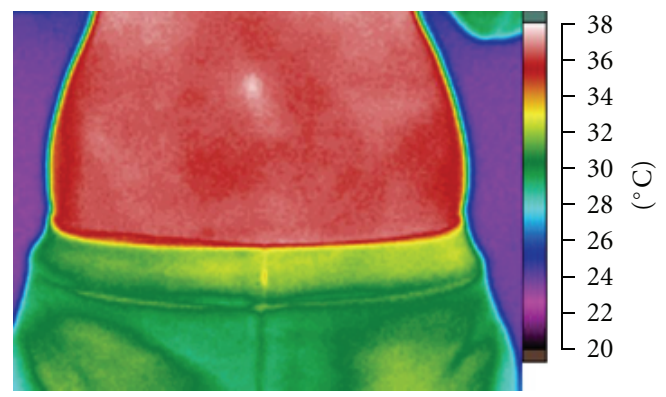

(b)

FIgURe 2: The abdominal skin temperature distribution of pretest (a) and posttest (b) of applying an FIR belt.

the whole day during menstruation, but could remove it in order to bathe. Application of an FIR belt or a control belt in sequence was also determined by random sampling (double-blind). The six precisely worded descriptions of pain degrees in the VRS (Chinese version) are scored as follows: none (0), very mild (1), mild (2), moderate (3), severe (4), and very severe (5). The 11-point NRS (Chinese version) is scored using eleven levels of pain, ranging from no pain (0) to the worst pain (10). For assessments of pain levels, there may be individual differences in subjects' feelings and cognition. Thus, this study adopted both the VRS and NRS as complementary indices so that participants' pain levels could be more precisely assessed.

2.5. Statistical Analysis. All data between groups before and after applying belts were analyzed by the Wilcoxon SignedRank Test using the SPSS 12.0 software package for statistical analysis. A value of $P<0.05$ was considered statistically significant $\left(^{*}\right)$ and $P<0.01$ was highly significant $(* *)$.

\section{Results and Discussion}

3.1. Abdominal Temperature. Figure 2 shows the results of temperature differences between pretreatment and posttreatment in the FIR belt group. There was a temperature increase after treatment which was probably due to the thermal insulation effect of the belt. The statistical analysis indicates that the average increases in temperature in the control group and FIR group were $0.36^{\circ} \mathrm{C}$ and $0.93^{\circ} \mathrm{C}$, respectively (Figure 3 ). The temperature increase in the FIR group was thus enhanced by $0.57^{\circ} \mathrm{C}$ compared with that found in the

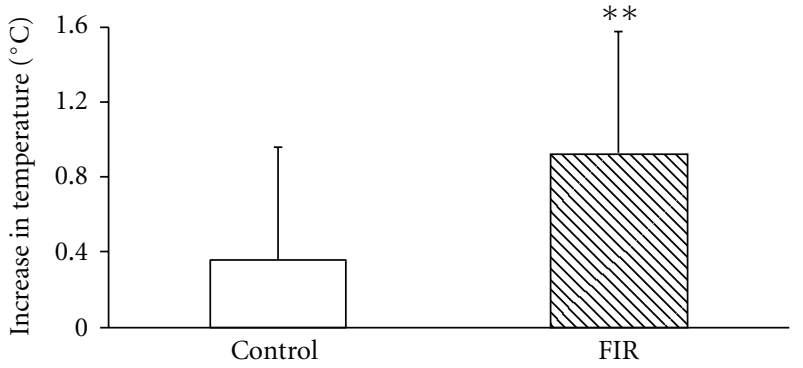

Figure 3: The average increases in temperature after wearing the control belt and FIR belt. The symbol ** indicates a highly significant difference between groups using the Wilcoxon SignedRank Test.

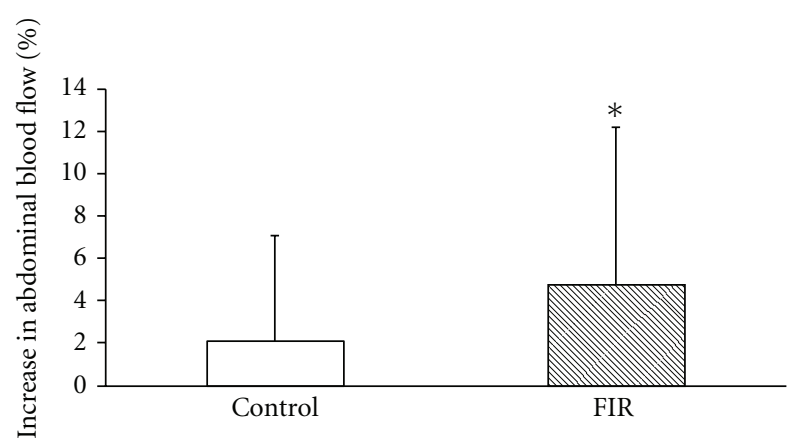

FIgURE 4: The average increase in abdominal blood flow after wearing the control belt and FIR belt. The symbol * indicates a significant difference between groups using the Wilcoxon SignedRank Test.

control group, which demonstrates that the FIR treatment was associated with a greater rise in abdominal temperature than the control condition. It is reasonable to conclude that the thermal insulation properties of the FIR belt resulted in an increase in the subjects' temperatures. This finding is consistent with previously reported results in similar studies [24-26].

3.2. Abdominal Blood Flow. With the rise in body temperature, there was a corresponding rise in abdominal blood flow after using the belts for 30 minutes. The average blood perfusion units measured by the MoorLDI2-IR Laser Doppler Imager were 116.5 and 119.0 before and after intervention in the control group, and 115.9 and 121.4 in the FIR group, respectively. There were increases in blood flow of $2.12 \%$ and $4.78 \%$ in the control group and FIR group, respectively (Figure 4). Thus, the increase in abdominal blood flow in the FIR group was enhanced by $2.66 \%$ compared with that in the control group.

3.3. VRS and NRS Scores. Figures 5 and 6 show the subjects' distribution of VRS and NRS scores on the first day of menstruation. The results of both VRS and NRS showed a general trend for lower scores in the FIR group. Compared to the blank group, the percentage of subjects with a VRS score above 3 was reduced from $27 \%$ to $10 \%$ and the percentage 


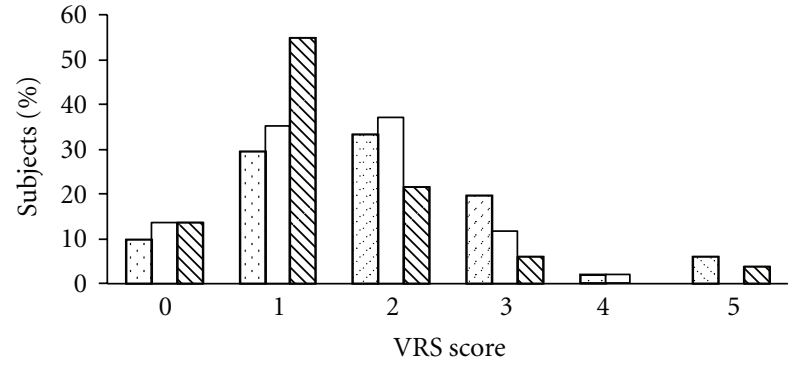

$$
\begin{aligned}
& \square \text { Blank group } \\
& \square \text { Control group } \\
& \square \text { FIR group }
\end{aligned}
$$

FIGURE 5: The distribution of subjects' VRS scores on the first day of menstruation.

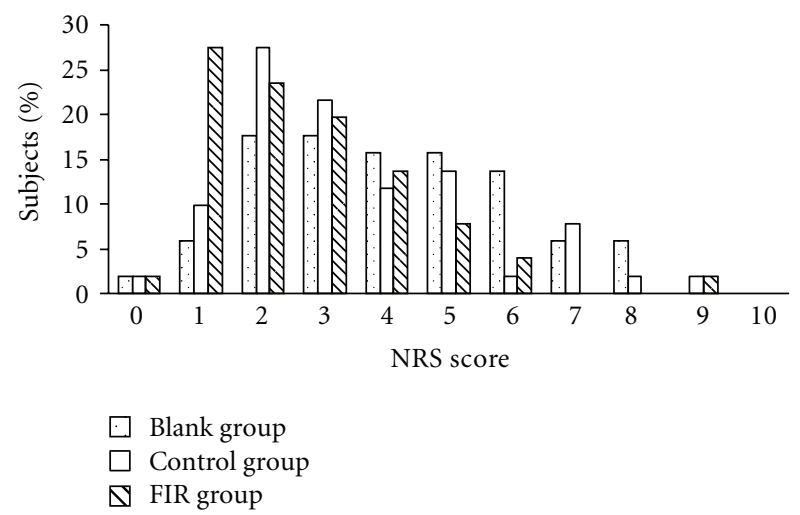

FIGURE 6: The distribution of subjects' NRS scores on the first day of menstruation.

of those with an NRS score above 5 was reduced from $41 \%$ to $14 \%$ in the FIR group. The distribution of scores in the control group was between those of the blank and FIR groups. Therefore, the placebo belt showed some efficacy for pain relief, but a better reduction in pain was reported by patients wearing the FIR belt.

The results of the second day (Figures 7 and 8) were similar to those found on the first day. After the belt intervention, pain intensity was reduced with respect to baseline. A greater reduction of pain was observed in the FIR belt group as compared with that in the control belt group. Compared with the blank group, the percentage of subjects with a VRS score above 3 was reduced from $14 \%$ to $2 \%$ (Figure 7) and the percentage of those with an NRS score above 5 was reduced from $35 \%$ to $12 \%$ (Figure 8 ) in the FIR group.

The results of the third day (Figures 9 and 10) were comparable to the results of the first and second days. The FIR group appeared to be the most efficient in terms of pain reduction. Compared to the blank group, the percentage of subjects with a VRS score above 3 was reduced from $10 \%$ to $2 \%$ (Figure 9) and the percentage of subjects with an NRS score above 5 was reduced from $14 \%$ to $6 \%$ (Figure 10) in the FIR group.

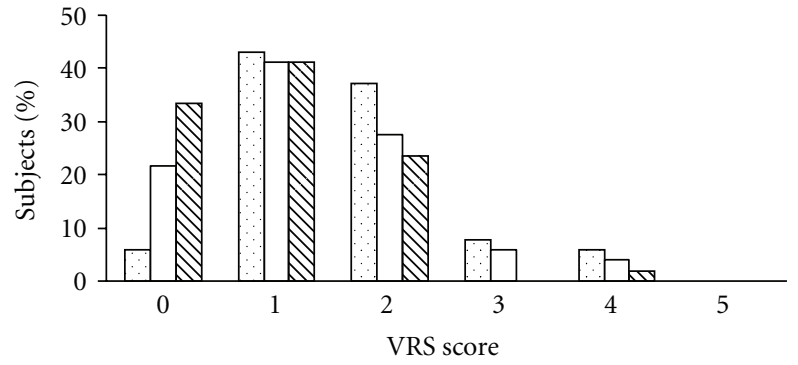

$$
\begin{aligned}
& \square \text { Blank group } \\
& \square \text { Control group } \\
& \triangle \text { FIR group }
\end{aligned}
$$

Figure 7: The distribution of subjects' VRS scores on the second day of menstruation.

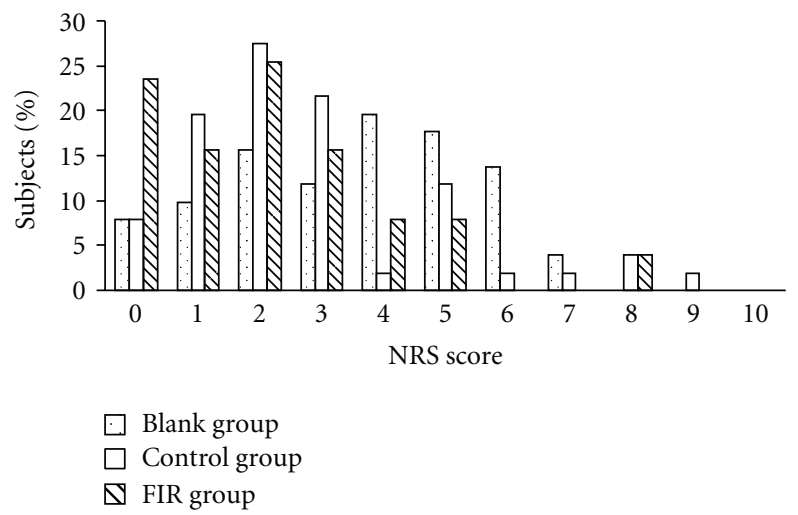

Figure 8: The distribution of subjects' NRS scores on the second day of menstruation.

Figures 11 and 12 are the box-and-whisker plots of VRS and NRS, respectively. The median levels of VRS and NRS in the FIR group were significantly lower than those of the blank group. The VRS and NRS scores in the FIR group showed highly significant differences compared with those of the control and blank groups. The scores in the control group also showed significant differences compared with those in the blank group on the second day (NRS) and the third day (VRS and NRS). Figure 13(a) shows the pain duration data and Figure 13(b) indicates their average and standard deviation. The lower and upper quartiles in the FIR group were significantly lower than those in the blank and control groups. Compared to the blank group, it can be seen that duration of pain in the FIR group was significantly decreased in the menstrual period from 2.4 to 1.8 days. However, there was no significant difference between the blank and control groups. Based on these findings, the authors speculate that the application of sufficient warmth to the belly region can improve blood circulation and shorten the menstrual pain duration.

Previous research has shown that the dysregulation of endometrial blood flow can cause menstrual disorders [35]. Strong and abnormal uterine contractions in women with primary dysmenorrhea can decrease uterine blood flow, 


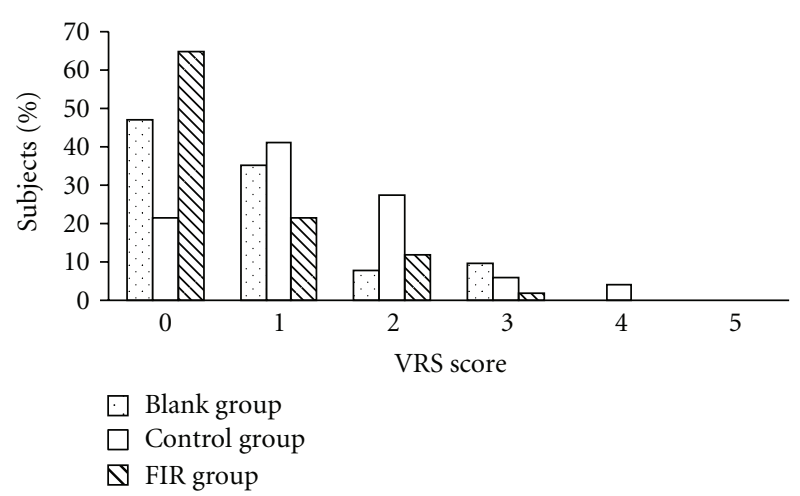

FIGURE 9: The distribution of subjects' VRS scores on the third day of menstruation.

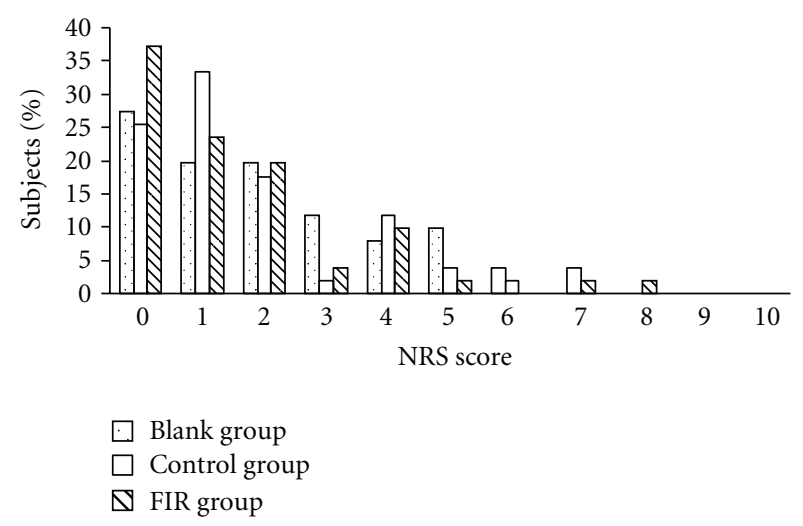

FIGURE 10: The distribution of subjects' NRS scores on the third day of menstruation.

resulting in pain in the uterus and the development of ischemia [4]. The application of FIR increases abdominal temperature and blood circulation and reduces muscle tension, leading to the relief of menstrual pain. Related studies also indicate that local heat treatment can alleviate dysmenorrhea [14].

In addition to the aforementioned thermal effects, FIR also exerts nonthermal effects in pain relief of dysmenorrhea. Previous studies showed that FIR could increase generation of nitric oxide (NO) and calmodulin in cells $[19,20,36]$. Associated physiological roles of NO include immune regulation [37, 38], neurotransmission [39], and vascular smooth muscle relaxation $[40,41]$. Previous reports have demonstrated that $\mathrm{NO}$ in endothelial cells plays an important role in regulating smooth muscle [42]. Endothelial NO in smooth muscle cells can activate guanylyl cyclase to produce cyclic guanosine monophosphate (cGMP). The cGMP sequentially activates protein kinase $G$, reduces smooth muscle intracellular calcium concentration, inhibits myosin light chain phosphorylation, and ultimately promotes smooth muscle relaxation [42-44]. NO has also been shown to help relax the uterus, thereby reducing the degree of dysmenorrhea [45].

Ischemia is mainly a consequence of decreased microcirculation or a reduction in local muscle blood flow. This scenario has important consequences for cellular metabolic

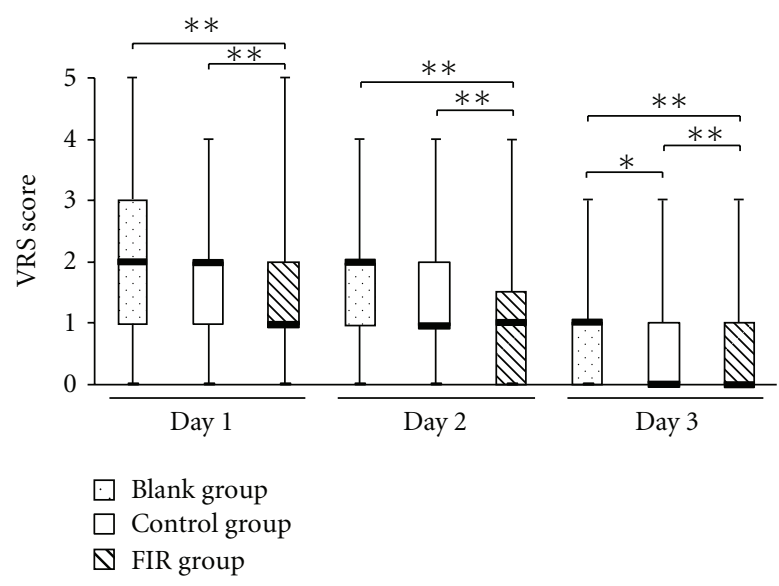

FIGURE 11: The VRS scores on the first three days of menstruation. The symbols * and ** indicate a significant and highly significant difference between groups using the Wilcoxon Signed-Rank Test, respectively.
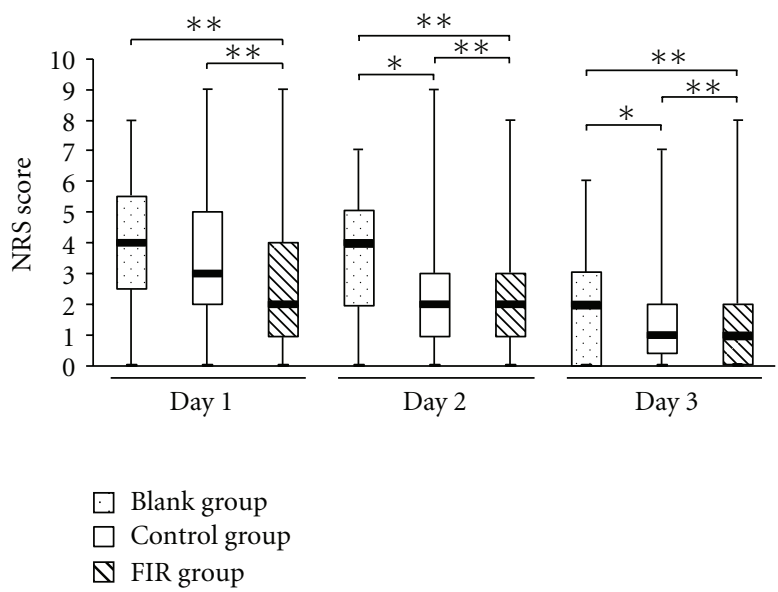

FIGURE 12: The NRS scores on the first three days of menstruation. The symbols $*$ and ${ }^{* *}$ indicate a significant and highly significant difference between groups using the Wilcoxon Signed-Rank Test, respectively.

status, as metabolic and acid-base status is significantly worsened with increased acidosis. During dysmenorrhea, the corresponding regional organic ischemia is associated with increased oxidative stress due to increased levels of reactive oxygen species (ROS), such as superoxide and hydrogen peroxide, which are responsible for destructive processes in organic tissues. We demonstrated that the FIR ceramic material exerted an antioxidant effect by increasing hydrogen peroxide-scavenging activity $[21,22]$.

We also found that FIR induced anti-inflammatory effects by inhibiting prostaglandin E2 (PGE2) [46], and FIR irradiation caused significant inhibition of COX-2 elevation during inflammation [47]. Prostaglandin synthesis is mediated primarily by cyclooxygenase (COX-1 and COX2 ), which catalyzes the metabolism of arachidonate to prostaglandin $\mathrm{H} 2$ and then produces PGE2 [48], stimulating 


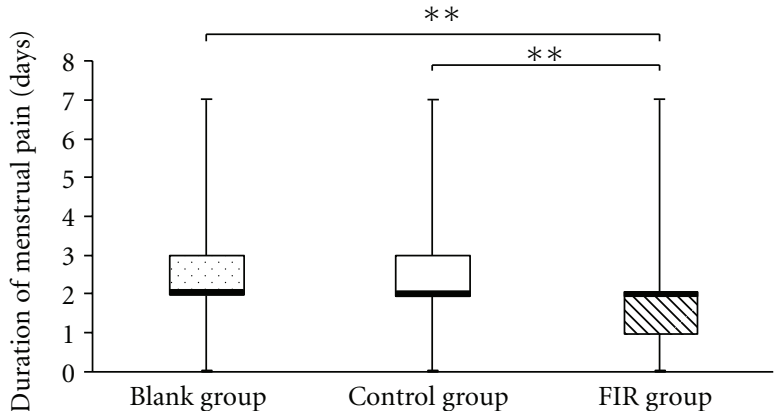

(a)

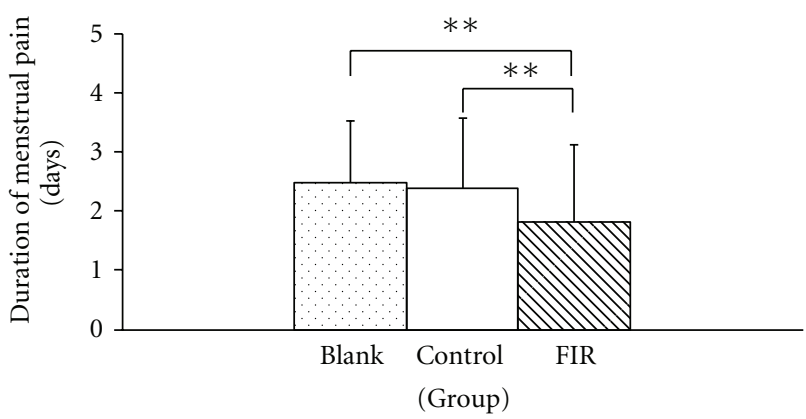

(b)

Figure 13: The duration of menstrual pain presented by (a) a boxand-whisker plot and (b) a bar chart indicating the average and standard deviation. The symbol ** indicates a highly significant difference between groups using the Wilcoxon Signed-Rank Test.

uterine contractility, which causes the pain. COX-2 and prostaglandin are strongly related to the severity of primary dysmenorrhea. Previous studies also reported that the main cause of primary dysmenorrhea is an abnormal increase in uterine prostaglandin level, leading to pain caused by uterine smooth muscle contraction [49]. Therefore, reducing PGE2 by applying FIR may lessen the discomfort caused by dysmenorrhea.

\section{Conclusions}

These findings of this study showed that the somatothermal FIR belt was effective as a novel alternative therapy for relief of primary dysmenorrhea. The FIR belt provided better therapeutic effects in terms of pain relief as well as greater elevation of skin temperature and promotion of blood circulation compared with those observed in patients wearing the sham belt. Compared with traditional complementary and alternative medicine therapies for pain reduction in patients with primary dysmenorrhea, our method has a number of advantages, which include convenience and ease of use, and no external energy source is required. We believe that this novel, noninvasive, and convenient FIR therapy may be of practical use in a clinical setting and suggest that further studies be conducted to confirm our results.

\section{Conflict of Interests}

The authors declare that they have no significant conflict of financial, professional, or personal interests.

\section{Acknowledgments}

This work was supported by a Grant from Taichung Veterans General Hospital/HungKuang University Joint Research Program (TCVGH-HK 998005). The authors also gratefully acknowledge the help of Roy Sun.

\section{References}

[1] L. French, "Dysmenorrhea," American Family Physician, vol. 71, no. 2, pp. 285-292, 2005.

[2] M. A. Burnett, V. Antao, A. Black et al., "Prevalence of primary dysmenorrhea in Canada," Journal of Obstetrics and Gynaecology Canada, vol. 27, no. 8, pp. 765-770, 2005.

[3] G. Eryilmaz and F. Ozdemir, "Evaluation of Menstrual Pain Management Approaches by Northeastern Anatolian Adolescents," Pain Management Nursing, vol. 10, no. 1, pp. 40-47, 2009.

[4] M. Y. Dawood, "Primary dysmenorrhea: advances in pathogenesis and management," Obstetrics and Gynecology, vol. 108, no. 2, pp. 428-441, 2006.

[5] J. Marjoribanks, M. Proctor, C. Farquhar, and R. S. Derks, "Nonsteroidal anti-inflammatory drugs for dysmenorrhoea," Cochrane Database of Systematic Reviews, no. 1, Article ID CD001751, 2010.

[6] M. L. Proctor, C. A. Smith, C. M. Farquhar, and R. W. Stones, "Transcutaneous electrical nerve stimulation and acupuncture for primary dysmenorrhoea," Cochrane Database of Systematic Reviews, no. 1, Article ID CD002123, 2002.

[7] H. M. Chen and C. H. Chen, "Effects of acupressure at the Sanyinjiao point on primary dysmenorrhoea," Journal of Advanced Nursing, vol. 48, no. 4, pp. 380-387, 2004.

[8] S. H. Han, M. H. Hur, J. Buckle, J. Choi, and M. S. Lee, "Effect of aromatherapy on symptoms of dysmenorrhea in college students: a randomized placebo-controlled clinical trial," Journal of Alternative and Complementary Medicine, vol. 12, no. 6, pp. 535-541, 2006.

[9] J. D. Adams and C. Garcia, "Women's health among the Chumash," Evidence-based Complementary and Alternative Medicine, vol. 3, no. 1, pp. 125-131, 2006.

[10] E. M. Jun, S. Chang, D. H. Kang, and S. Kim, "Effects of acupressure on dysmenorrhea and skin temperature changes in college students: a non-randomized controlled trial," International Journal of Nursing Studies, vol. 44, no. 6, pp. 973$981,2007$.

[11] N. Tugay, T. Akbayrak, F. Demirtürk et al., "Effectiveness of transcutaneous electrical nerve stimulation and interferential current in primary dysmenorrhea," Pain Medicine, vol. 8, no. 4, pp. 295-300, 2007.

[12] J. F. Cheng, Z. Y. J. Lu, Y. C. Su, L. C. Chiang, and R. Y. Wang, "A traditional Chinese herbal medicine used to treat dysmenorrhoea among Taiwanese women," Journal of Clinical Nursing, vol. 17, no. 19, pp. 2588-2595, 2008.

[13] V. Iorno, R. Burani, B. Bianchini, E. Minelli, F. Martinelli, and S. Ciatto, "Acupuncture treatment of dysmenorrhea resistant to conventional medical treatment," Evidence-Based 
Complementary and Alternative Medicine, vol. 5, no. 2, pp. 227-230, 2008.

[14] M. D. Akin, K. W. Weingand, D. A. Hengehold, M. B. Goodale, R. T. Hinkle, and R. P. Smith, "Continuous low-level topical heat in the treatment of dysmenorrhea," Obstetrics and Gynecology, vol. 97, no. 3, pp. 343-349, 2001.

[15] S. F. Nadler, D. J. Steiner, G. N. Erasala, D. A. Hengehold, S. B. Abeln, and K. W. Weingand, "Continuous low-level heatwrap therapy for treating acute nonspecific low back pain," Archives of Physical Medicine and Rehabilitation, vol. 84, no. 3, pp. 329334, 2003.

[16] C. A. Smith, C. A. Crowther, O. Petrucco, J. Beilby, and H. Dent, "Acupuncture to treat primary dysmenorrhea in women: a randomized controlled trial," Evidence-Based Complementary and Alternative Medicine, vol. 2011, Article ID 612464, 11 pages, 2011.

[17] D. Taylor, C. Miaskowski, and J. Kohn, "A randomized clinical trial of the effectiveness of an acupressure device (Relief Brief) for managing symptoms of dysmenorrhea," Journal of Alternative and Complementary Medicine, vol. 8, no. 3, pp. 357-370, 2002.

[18] S. Y. Yu, J. H. Chiu, S. D. Yang, Y. C. Hsu, W. Y. Lui, and $\mathrm{C} . \mathrm{W}$. Wu, "Biological effect of far-infrared therapy on increasing skin microcirculation in rats," Photodermatology Photoimmunology and Photomedicine, vol. 22, no. 2, pp. 7886, 2006.

[19] T. K. Leung, C. M. Lee, M. Y. Lin et al., "Far infrared ray irradiation induces intracellular generation of nitric oxide in breast cancer cells," Journal of Medical and Biological Engineering, vol. 29, no. 1, pp. 15-18, 2009.

[20] T. K. Leung, Y. S. Lin, Y. C. Chen et al., "Immunomodulatory effects of far-infrared ray irradiation via increasing calmodulin and nitric oxide production in raw 264.7 macrophages," Biomedical Engineering, vol. 21, no. 5, pp. 317-323, 2009.

[21] T. K. Leung, H. F. Shang, D. C. Chen et al., "Effects of far infrared rays on hydrogen peroxide-scavenging capacity," Biomedical Engineering, vol. 23, no. 2, pp. 99-105, 2011.

[22] T. K. Leung, Y. S. Lin, C. M. Lee et al., "Direct and indirect effects of ceramic far infrared radiation on the hydrogen peroxide-scavenging capacity and on murine macrophages under oxidative stress," Journal of Medical and Biological Engineering, vol. 31, no. 5, pp. 345-351, 2011.

[23] T. K. Leung, C. F. Chan, P. S. Lai, C. H. Yang, C. Y. Hsu, and Y. S. Lin, "Inhibitory effects of far-infrared irradiation generated by ceramic material on murine melanoma cell growth," International Journal of Photoenergy, vol. 2012, Article ID 646845, 8 pages, 2012.

[24] B. H. Yoo, C. M. Park, T. J. Oh, S. H. Han, H. H. Kang, and I. S. Chang, "Investigation of jewelry powders radiating farinfrared rays and the biological effects on human skin," Journal of Cosmetic Science, vol. 53, no. 3, pp. 175-184, 2002.

[25] G. D. Ko and D. Berbrayer, "Effect of ceramic-impregnated "thermoflow" gloves on patients with Raynaud's syndrome: randomized, placebo-controlled study," Alternative Medicine Review, vol. 7, no. 4, pp. 327-335, 2002.

[26] M. H. Shim, C. H. Park, and H. S. Shim, "Effect of ceramics on the physical and thermo-physiological performance of warmup suit," Textile Research Journal, vol. 79, no. 17, pp. 15571564, 2009.

[27] H. Toyokawa, Y. Matsui, J. Uhara et al., "Promotive effects of far-infrared ray on full-thickness skin wound healing in rats," Experimental Biology and Medicine, vol. 228, no. 6, pp. 724729, 2003.
[28] J. F. Tsai, S. Hsiao, and S. Y. Wang, "Infrared irradiation has potential antidepressant effect," Progress in Neuro-Psychopharmacology and Biological Psychiatry, vol. 31, no. 7, pp. 1397-1400, 2007.

[29] J. Ishibashi, K. Yamashita, T. Ishikawa et al., "The effects inhibiting the proliferation of cancer cells by far-infrared radiation (FIR) are controlled by the basal expression level of heat shock protein (HSP) 70A," Medical Oncology, vol. 25, no. 2, pp. 229-237, 2008.

[30] C. H. Lee, J. W. Roh, C. Y. Lim, J. H. Hong, J. K. Lee, and E. G. Min, "A multicenter, randomized, double-blind, placebo-controlled trial evaluating the efficacy and safety of a far infrared-emitting sericite belt in patients with primary dysmenorrhea," Complementary Therapies in Medicine, vol. 19, no. 4, pp. 187-193, 2011.

[31] B. Y. Liau, T. K. Leung, M. C. Ou, C. K. Ho, A. Yang, and Y. S. Lin, "Inhibitory effects of far-infrared ray emitting belts on primary dysmenorrhea," International Journal of Photoenergy, vol. 2012, Article ID 238468, 6 pages, 2012.

[32] A. M. Hug, T. Schmidts, J. Kuhlmann, D. Segger, G. Fotopoulos, and J. Heinzerling, "Skin hydration and cooling effect produced by the Voltaren vehicle gel," Skin Research and Technology, vol. 18, no. 2, pp. 199-206, 2011.

[33] A. Fauconnier, E. Dallongeville, C. Huchon, Y. Ville, and B. Falissard, "Measurement of acute pelvic pain intensity in gynecology: a comparison of five methods," Obstetrics and Gynecology, vol. 113, no. 2, pp. 260-269, 2009.

[34] C. Brunelli, E. Zecca, C. Martini et al., "Comparison of numerical and verbal rating scales to measure pain exacerbations in patients with chronic cancer pain," Health and Quality of Life Outcomes, vol. 8, article 42, 2010.

[35] N. J. Raine-Fenning, B. K. Campbell, N. R. Kendall, J. S. Clewes, and I. R. Jonhson, "Quantifying the changes in endometrial vascularity throughout the normal menstrual cycle with three-dimensional power Doppler angiography," Human Reproduction, vol. 19, no. 2, pp. 330-338, 2004.

[36] Y. H. Hsu, Y. C. Chen, T. H. Chen et al., "Far-infrared therapy induces the nuclear translocation of PLZF which inhibits VEGF-induced proliferation in human umbilical vein endothelial cells," PloS ONE, vol. 7, no. 1, Article ID e30674, 2012.

[37] A. Contestabile, "Targeting nitric oxide for tumor therapy," Current Pharmaceutical Design, vol. 16, no. 4, pp. 378-380, 2010.

[38] D. K. Ghosh, K. Ray, A. J. Rogers, N. J. Nahm, and J. C. Salerno, "FMN fluorescence in iNOS constructs reveals a series of conformational states involved in the reductase catalytic cycle," Federation of European Biochemical Societies Journal, vol. 279, no. 7, pp. 1306-1317, 2012.

[39] K. U. Domek-Łopacińska and J. B. Strosznajder, "Cyclic GMP and nitric oxide synthase in aging and alzheimer's disease," Molecular Neurobiology, vol. 41, no. 2-3, pp. 129-137, 2010.

[40] I. H. Wei, H. C. Tu, C. C. Huang, M. H. Tsai, C. Y. Tseng, and J. Y. Shieh, "(-)-Epigallocatechin gallate attenuates NADPH$\mathrm{d} / \mathrm{nNOS}$ expression in motor neurons of rats following peripheral nerve injury," BMC Neuroscience, vol. 12, article 52, 2011.

[41] B. Nossaman, E. Pankey, and P. Kadowitz, "Stimulators and activators of soluble guanylate cyclase: review and potential therapeutic indications," Critical Care Research and Practice, vol. 2012, Article ID 290805, 12 pages, 2012.

[42] K. H. Yuill, A. J. McNeish, Y. Kansui, C. J. Garland, and K. A. Dora, "Nitric oxide suppresses cerebral vasomotion by sgcindependent effects on ryanodine receptors and voltage-gated 
calcium channels," Journal of Vascular Research, vol. 47, no. 2, pp. 93-107, 2010.

[43] J. L. Zweier, C. A. Chen, and L. J. Druhan, "S-glutathionylation reshapes our understanding of endothelial nitric oxide synthase uncoupling and nitric oxide/reactive oxygen speciesmediated signaling," Antioxidants and Redox Signaling, vol. 14, no. 10, pp. 1769-1775, 2011.

[44] J. Kopincová, A. Púzserová, and I. Bernátová, "Biochemical aspects of nitric oxide synthase feedback regulation by nitric oxide," Interdisciplinary Toxicology, vol. 4, no. 2, pp. 63-68, 2011.

[45] H. P. Zahradnik, A. Hanjalic-Beck, and K. Groth, "Nonsteroidal anti-inflammatory drugs and hormonal contraceptives for pain relief from dysmenorrhea: a review," Contraception, vol. 81, no. 3, pp. 185-196, 2010.

[46] T. K. Leung, C. H. Chen, C. H. Lai et al., "Bone and joint protection ability of ceramic material with biological effects," Chinese Journal of Physiology, vol. 55, no. 1, pp. 47-54, 2012.

[47] T. K. Leung, Y. C. Liu, C. H. Chen, H. N. Fang, K. C. Chen, and C. M. Lee, "In vitro cell study of the possible anti-inflammatory and pain relief mechanism of far-infrared ray emitting ceramic material (BIOCERAMIC)," Journal of Medical and Biological Engineering. In press.

[48] M. A. Fortier, K. Krishnaswamy, G. Danyod, S. BoucherKovalik, and P. Chapdelaine, "A postgenomic integratred view of prostaglandins in reproduction: implications for other body systems," Journal of Physiology and Pharmacology, vol. 59, no. 1, pp. 65-89, 2008.

[49] T. Hosono, Y. Takashima, Y. Morita et al., "Effects of a heatand steam-generating sheet on relieving symptoms of primary dysmenorrhea in young women," Journal of Obstetrics and Gynaecology Research, vol. 36, no. 4, pp. 818-824, 2010. 


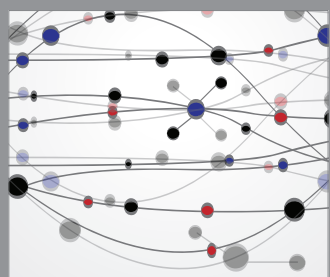

The Scientific World Journal
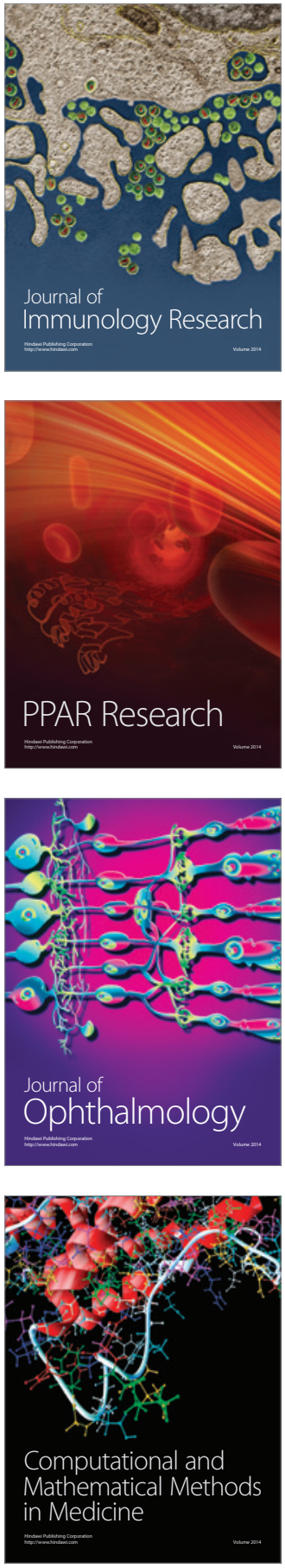

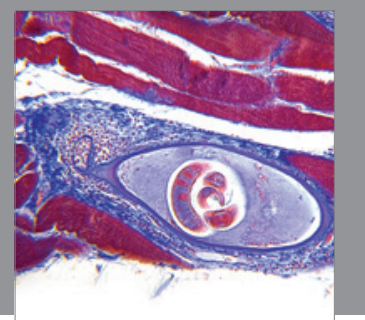

Gastroenterology

Research and Practice
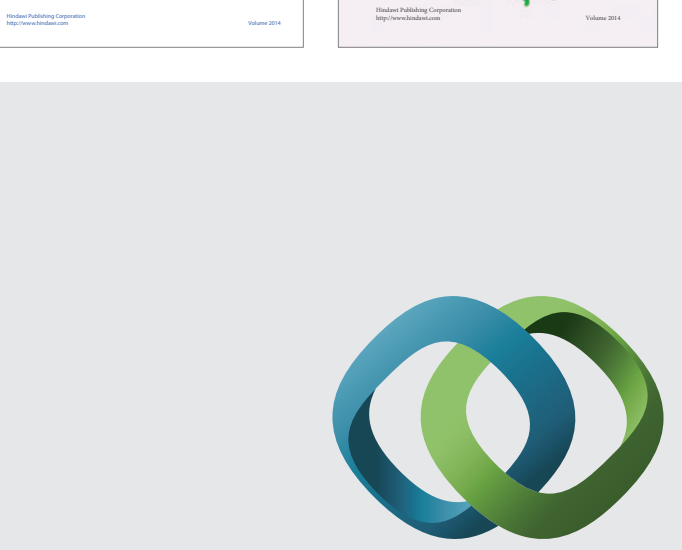

\section{Hindawi}

Submit your manuscripts at

http://www.hindawi.com
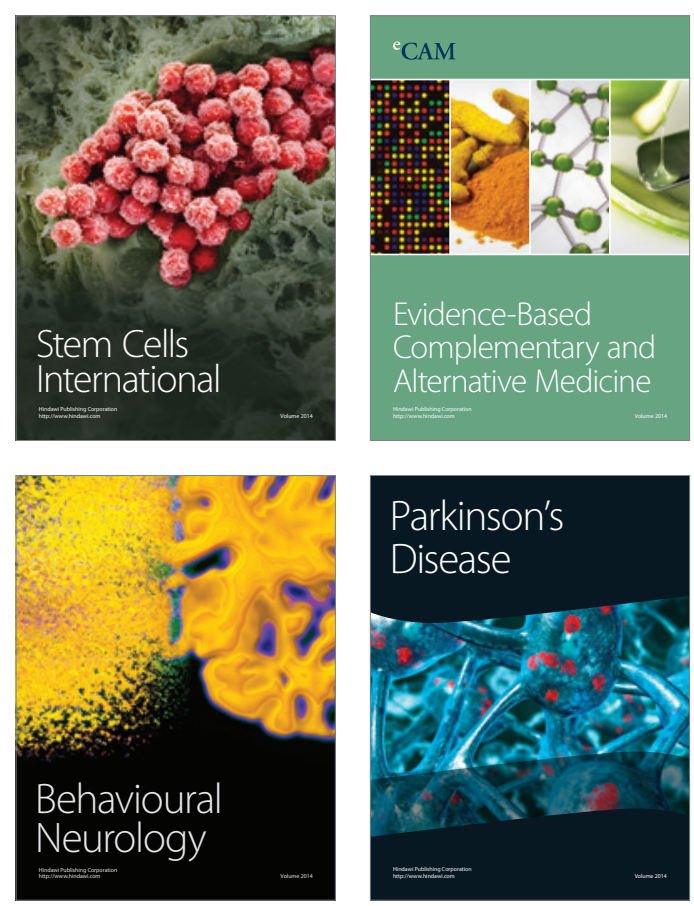

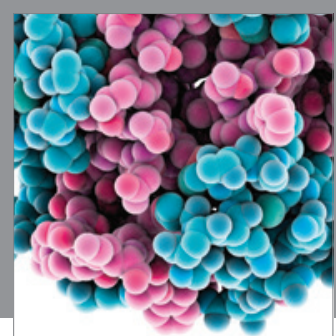

Journal of
Diabetes Research

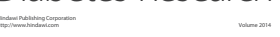

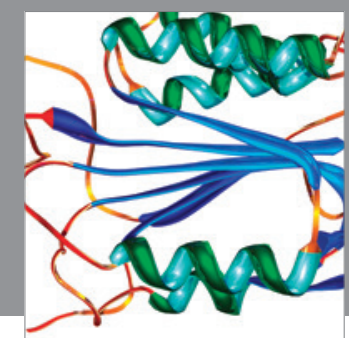

Disease Markers
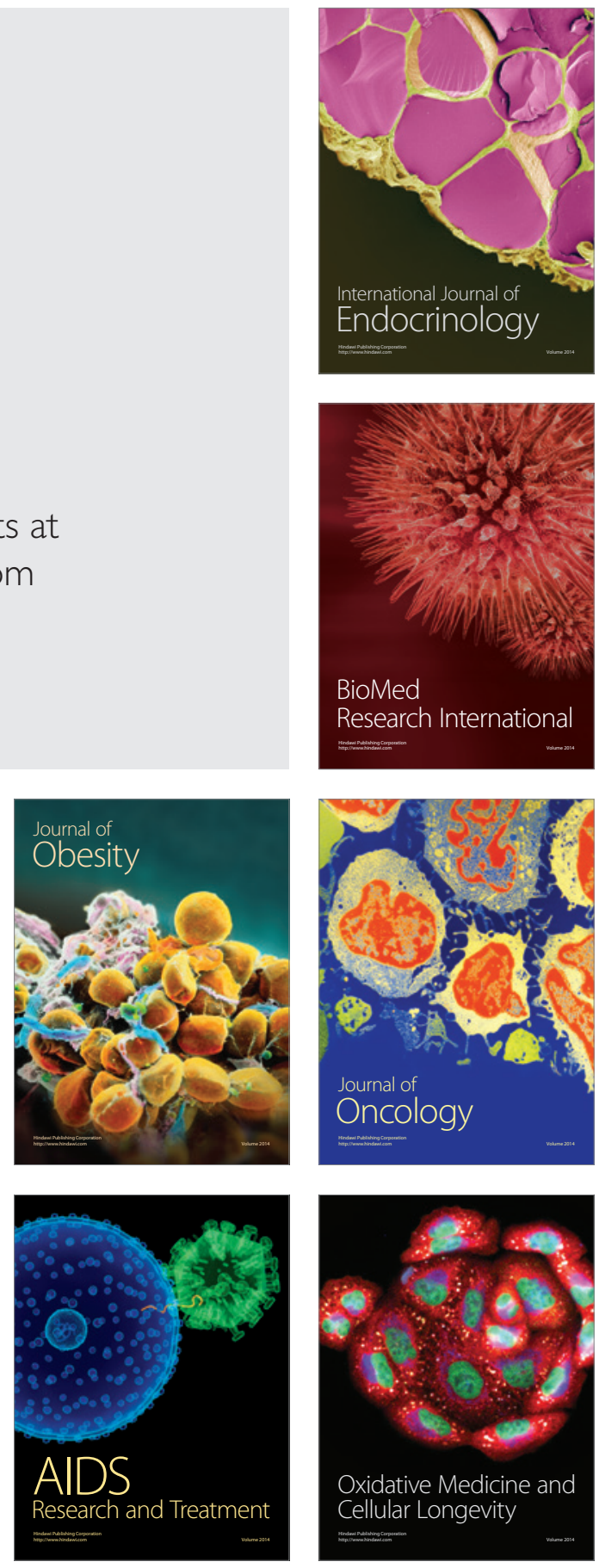